\title{
Different Selection Strategies for the Improvement of the Growth Performance and Carcass Traits of Japanese Quails
}

\section{-Author(s)}

Ahmad S'
Mehmood S'
Javed K"
Mahmud A'
Usman $\mathrm{A}^{\prime}$
Rehman $\mathrm{A}^{\prime}$
Ishaq HM"I'
Hussain J'
Ghayas A'

Department of Poultry Production, Faculty of Animal Production and Technology, University of Veterinary and Animal Sciences, Lahore, 54000, Pakistan.

" Department of Livestock Production, Faculty of Animal Production and Technology, University of Veterinary and Animal Sciences, Lahore, 54000, Pakistan.

III Department of Livestock and Poultry Production, Faculty of Veterinary Science, Bahauddin Zakariya University, Multan, 60000, Pakistan

\section{nMail Address}

Corresponding author e-mail address Sohail Ahmad

Department of Poultry Production, Faculty of Animal Production and Technology,

University of Veterinary and Animal

Sciences, Lahore-Pakistan.

Phone: +92-313-4288381

Email: sohail.ahmad@uvas.edu.pk

\section{EKeywords}

Pedigree Selection, Mass Selection, Parental Age groups, Close-bred Flocks, Feed Conversion Ratio, Carcass yield.

\section{ABSTRACT}

The present study evaluated different selection strategies to improve the growth performance and carcass traits of Japanese quails. To this end, 540 Japanese quails previously selected for high body weight at three ages (10, 12 and 14 weeks) from four close-bred flocks (Major, Kaleem, Saadat and Zahid) were subjected to three selection strategies: pedigree-based, mass selection or random-bred (controls). For pedigreebased selection, 108 birds (4 close-bred flocks $\times 3$ age groups $\times 9$ replicates $\times 1$ bird each) with full pedigree and selected for high body weight were used, whereas in mass selection 324 birds (4 close-bred flocks $\times 3$ age groups $\times 9$ replicates $\times 3$ birds each) with high body weight were selected to be the parents of next generation. Randombred controls included 108 birds (4 close-bred flocks $\times 3$ age groups $\times 9$ replicates $\times 1$ bird each) which were not previously submitted to selection. The effects of selection strategies of Japanese quail parents from four close-bred flocks (CBF) at three ages on the performance growth and carcass traits of their progenies were measured. The parent flocks were selected at three ages (10,12 and 14 weeks) and reared for 20 weeks, and their progenies were reared for four weeks. The progeny of pedigree-based selected parents presented better feed intake (g), body weight gain (g), feed conversion ratio, live and carcass weights (g), and carcass yield. The progeny of 10- and 14-week-old parents showed better growth performance and carcass traits, respectively. Moreover, there was no influence of $\mathrm{CBF}$ on growth and carcass traits. It was concluded that pedigree-based selection had pronounced effect on the growth performance and carcass traits of the progeny compared with mass selection and random breeding. It is suggested that parental ages of 10 and 14 weeks of age promote better progeny growth performance and carcass traits.

\section{INTRODUCTION}

Selection is a basic tool to exploit and improve the productive potential of birds. Different selection strategies have been working throughout the world comprising mass selection to fully pedigree selection (Marks, 1991, 1996; Minvielle et al., 1997; Narinç et al., 2016; Durmuş et al., 2017). Selection programs remarkably improve economic traits in broilers with2.4-fold improvements in growth rate and carcass traits; however, feed conversion ratio has improved only $0.025 \%$ per year.

In Japanese quails, genetic improvements of $9.6,8.8$ and $8.2 \mathrm{~g}$ in body weight at four weeks of age (4-wk BW) were observed in generations 2, 3 and 4, respectively (Varkoohi et al., 2010). Over the same period of selection for 4-wk BW, the improvement in feed conversion ratio (FCR) was 0.16 units. These results indicate that selection for reduced FCR 
Ahmad S, Mehmood S, Javed K, Mahmud A, Usman M, Rehman A, Ishaq HM, Hussain J, Ghayas A

\section{Different Selection Strategies for the Improvement of the Growth Performance and Carcass Traits of Japanese Quails}

increases body weight and weight gain and reduces feed intake and residual feed intake as correlated responses (Varkoohi et al., 2010).

Moreover, a strong genetic correlation between 4-wk BW and carcass traits was recorded (Khaldari et al., 2010). Another study reported that slaughter age significantly influenced the carcass traits of Japanese quails (Narinc et al., 2014). The findings of Tukmut et al. (1999) revealed that slaughter weight, carcass weight, carcass yield, abdominal fat weight, organ weight and organ yields of female quails were affected by selection. Carcass weight, carcass percent, breast weight and thigh percent were significantly affected by Japanese quail strain (Vali et al., 2005)

Selection experiments provide background knowledge of the inheritance of complex traits and allow the evaluation of the hypothetical predictions by comparing observations against expectations (Szwaczkowski, 2003). The selection objectives may be short-termed, i.e., using genetic additive variance and covariance test to estimate genetic response or long-termed to measure the selection response or additive variance caused by the selection procedure (Hill \& Mackay, 2004). Long-term response to selection is more focused on fixing the probabilities of alleles responsible for the trait(s) under consideration and it is generally agreed that to achieve most of the goals of long term selection more than thirty generations will be required (Reddy, 1996). On the other hand, shortterm selection response might be attributed to alleles segregating in the population (Fuller et al., 2005).

Japanese quails have called significant attention in recent years due to their high resistance to diseases as well as for its use for meat and egg production. They mature at an early age of 6 weeks and females are usually in full production by about 8 weeks of age (Ashok \& Prabakaran, 2012). Due to their faster growth rate and high egg production, quails may be an interesting option to study production, reproduction, egg quality traits, as well as metabolic and immunological traits. Moreover, their generation interval is short, of almost 3-4 generations per year, and present high recombination rate, which makes of Japanese quails a very suitable species for genetic studies (Burt \& Pourquie, 2003).

The profitability of quail enterprises largely depends on carcass quality, and many research studies have been conducted to describe quail carcass traits (Odunsi \& Kehinde, 2009). Among these, carcass yield is considered as trait of interest and in conventional selection experiments, quail can serve as the best avian model due to several reasons, including that they are relatively easy to handle and have cheap maintenance costs (Daikwao et al., 2013).

Despite having enormous potential, very little research has been conducted on the acclimatization of Japanese quails to Pakistan's environment. Keeping above in view, Japanese quail parents from four closebred flocks of three ages were subjected to three selection strategies and the growth performance and carcass traits of their progenies were evaluated.

\section{MATERIALS AND METHODS}

The present study was conducted at Avian Research and Training (ART) Centre, Department of Poultry Production, University of Veterinary and Animal Sciences, Lahore, Pakistan, to evaluate effect of the selection for higher body weight at 4 weeks of age of 4 close-bred flocks of three age groups of Japanese quails during $4^{\text {th }}$ generation.

A total of 540 quail breeders previously selected for 4-wk BW were subjected to selective breeding. Birds were distributed according to completely randomized design in a $3 \times 4 \times 3$ factorial arrangement. The treatments consisted of three selection strategies (pedigree-based, mass-selected, and random-bred), four close-bred flocks (Major, Kaleem, Saadat \& Zahid; Jatoi et al., 2013) and three parental age groups (10, 12 and 14 weeks), totaling 36 treatments with nine replicates or 324 experimental units.

The parent flocks were selected at three ages (10, 12 and 14 weeks) and reared for 20 weeks a density of $220 \mathrm{~cm}^{2} / \mathrm{bird}$ ); however, their progenies were reared under standard managemental conditions during the four experimental weeks $\left[83.61 \mathrm{~cm}^{2} /\right.$ bird during brooding (1-14 $\mathrm{d}$ of age) and $150 \mathrm{~cm}^{2} /$ bird during the growing phase (15-28 d of age)], as previously described by Narinc et al. (2013a).

\section{Selection Procedures}

Birds were divided in three main groups according to selection strategies, i.e., pedigree-based, massselected and random-bred groups, with 108, 324, and 108 birds, respectively. In first group, quails (4 close-bred flock $\times 3$ age groups $\times 9$ replicates $\times 1$ bird each) having higher body weight with full pedigree record were selected to draw comparison with birds subjected to mass selection and random-bred group. In second group, quails (4 close-bred flock $\times 3$ age groups $\times 9$ replicates $\times 3$ bird each) were subjected to mass selection in which birds with the highest body 
Ahmad S, Mehmood S, Javed K, Mahmud A, Usman M, Rehman A, Ishaq HM, Hussain J, Ghayas A

\section{Different Selection Strategies for the Improvement of the Growth Performance and Carcass Traits of Japanese Quails}

weight were selected to be the parents of the next generation. In the third group, quails (4 close-bred flock $\times 3$ age groups $\times 9$ replicates $\times 1$ bird each) were randomly selected and were considered the control group (Hussain et al., 2013).

\section{Housing and Management}

All experimental birds were maintained in wellventilated octagonal quail house $\left(21.68 \mathrm{~m}^{2}\right)$ equipped with multi-deck cages specially designed for separate quail rearing and breeding. The breeder quails were reared for 20 weeks (provided with 16 hours light). Meat-type quails were reared for 4 weeks and provided with 24 hours light. During brooding (first two weeks) 25 lux were provided and light intensity was decreased up to 5-10lux during the rearing period of two weeks (Narinc et al., 2013b). Two feeds were formulated as recommended by the NRC standards (1994): one for breeders (20\% CP and $2900 \mathrm{kcal} \mathrm{ME} / \mathrm{kg}$ ) and one for meat-type quails (24\% CP and $2900 \mathrm{kcal} \mathrm{ME} / \mathrm{kg}$ ). The nipple drinker system ensured the availability of clean drinking water. In case of pedigreed and randombred control birds, one individual female was kept per replicate, whereas in mass-selected birds, three females were kept per replicate. The stud-mating system was performed in pedigreed and random-bred control groups, whereas pen-mating system was practiced in mass-selected groups.

Growth performance parameters were determined at 4 weeks of age. Feed intake ( $\mathrm{g}$ ) was record as average feed intake/ bird /day and converted into weekly basis for the calculation of weekly feed conversion ratio. Feed intake (g) was calculated as feed offer (g) feed residue (g). Body weight gain (g) was calculated by subtracting final from initial body weight. Feed conversion ratio (FCR) was calculated on weekly basis by the following formula:

$$
\mathrm{FCR}=\frac{\text { Feedintake }(\mathrm{g})}{\text { Body weight gain }(\mathrm{g})}
$$

Mortality (\%)recorded on daily basis (if any) and converted into weekly basis to calculate mortality \% as:

$$
\text { Mortality }=\frac{\text { Dead Birds }}{\text { Total Birds }} \times 100
$$

At the end of $4^{\text {th }}$ week, three birds per replicate were slaughtered and following parameters were recorded. To record live weight (g), each bird was weighed on a digital scale to the nearest $0.5 \mathrm{~g}$. Birds were slaughtered, and carcass weight $(\mathrm{g})$ was determined after plucking and evisceration on a digital scale to the nearest 0.5 g. Carcass yield, liver, gizzard and heart weights were calculated relative to body weight and expressed in percentages, according to following formula:

$$
\frac{\text { Carcass }}{\text { parts }} \text { yield }(\%)=\frac{\frac{\text { carcass }}{\text { parts }} \text { weight }(\mathrm{g})}{\text { Livebody weight at slaughter }(\mathrm{g})} \times 100
$$

\section{Statistical Analysis}

Data were analyzed by analysis of variance using the PROC MIXED of SAS software (version 9.1). Means were compared by Tukey's HSD test, assuming the following mathematical model:

$Y_{i j k l}=\mu+S_{i}+A_{j}+C_{k}+(S A)_{i j}+(S C)_{i j k}+(A C)_{j k}+\varepsilon_{i j k l}$

Where,

$Y_{\mathrm{ijkl}}=$ Dependent variable

$\mu=$ Overall population mean

$\mathrm{S}_{\mathrm{i}}=$ Effect of $\mathrm{i}^{\text {th }}$ Selection Strategy assumed as fixed effect ( $i=1,2,3$; Pedigree, Mass-selected, Randombred Control)

$A=$ Effect of $j^{\text {th }}$ Parental Age group assumed as fixed effect ( $\mathrm{j}=$ week $1,2,3 ; 10,12$ and 14)

$C_{k}=$ Effect of $k^{\text {th }}$ Close-bred Flock assumed as random effect ( $k=1,2,3,4$; Major, Kaleem, Sadaat \& Zahid)

$(\mathrm{SA})_{\mathrm{ij}}=$ Interaction effect between selection strategy and parental age groups

$(\mathrm{SC})_{i k}=$ Interaction effect between selection strategy and close-bred flock

$(A C)_{j k}=$ Interaction effect between parental age groups and close-bred flock

$\varepsilon_{\mathrm{ijkl}}=$ Residual error associated with $\mathrm{ith}^{\text {th }}, \mathrm{j}^{\text {th }}$ and $\mathrm{k}^{\text {th }}$ treatment NID $\sim 0, \sigma^{2}$

\section{RESULTS AND DISCUSSION}

\section{Growth Performance}

Means and standard errors of cumulative feed intake (g), weight gain (g), FCR and mortality\% are presented in Table 1. Significantly $(p \leq 0.05)$ higher feed intake was observed in pedigree birds $(433.13 \pm 0.77$ g) compared with random-bred $(429.36 \pm 0.80 \mathrm{~g})$ and mass-selected $(428.90 \pm 0.51 \mathrm{~g})$ birds. However, no significant effects of CBF or parental ages were observed on feed intake in present study; however, a significant interaction of selection strategies interact with $\mathrm{CBF}$ and parental age were observed for cumulative feed intake. The significantly higher feed intake of pedigree birds compared with random-bred 
Ahmad S, Mehmood S, Javed K,

Mahmud A, Usman M, Rehman A,

Ishaq HM, Hussain J, Ghayas A

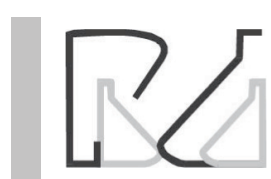

Different Selection Strategies for the Improvement of the Growth Performance and Carcass Traits of Japanese Quails

Table 1 - Effect of selection strategies of parent Japanese quails from four close-bred flocks of three different age on the growth performance of their progenies.

\begin{tabular}{|c|c|c|c|c|}
\hline & Cum. FI & Cum. Gain & Cum. FCR & Mortality \\
\hline & \multicolumn{2}{|c|}{----------(g)---------- } & & ---(\%)--- \\
\hline \multicolumn{5}{|l|}{ Selection Strategies } \\
\hline Pedigree & $433.13 \pm 0.77^{a}$ & $182.19 \pm 0.79^{a}$ & $2.38 \pm 0.01^{b}$ & $12.12 \pm 0.07$ \\
\hline Mass Selection & $428.90 \pm 0.51^{b}$ & $179.96 \pm 0.50^{b}$ & $2.39 \pm 0.01^{b}$ & $12.19 \pm 0.07$ \\
\hline Random-bred & $429.36 \pm 0.80^{b}$ & $175.57 \pm 0.76^{c}$ & $2.45 \pm 0.01^{a}$ & $12.21 \pm 0.06$ \\
\hline \multicolumn{5}{|l|}{ Close-bred Flocks } \\
\hline Major & $429.59 \pm 0.74$ & $179.30 \pm 0.96$ & $2.40 \pm 0.01$ & $12.30 \pm 0.10$ \\
\hline Kaleem & $431.71 \pm 0.65$ & $179.56 \pm 0.94$ & $2.41 \pm 0.01$ & $12.09 \pm 0.06$ \\
\hline Saadat & $430.32 \pm 0.91$ & $178.39 \pm 0.72$ & $2.42 \pm 0.01$ & $12.16 \pm 0.05$ \\
\hline Zahid & $430.21 \pm 1.01$ & $179.70 \pm 0.76$ & $2.40 \pm 0.01$ & $12.15 \pm 0.09$ \\
\hline \multicolumn{5}{|l|}{ Parental Age Groups } \\
\hline 10 weeks & $430.87 \pm 0.70$ & $180.81 \pm 0.81^{a}$ & $2.39 \pm 0.01^{b}$ & $12.17 \pm 0.06$ \\
\hline 12 weeks & $429.59 \pm 0.80$ & $179.40 \pm 0.66^{\mathrm{ab}}$ & $2.40 \pm 0.01^{b}$ & $12.13 \pm 0.06$ \\
\hline 14 weeks & $430.93 \pm 0.67$ & $177.52 \pm 0.71^{b}$ & $2.43 \pm 0.01^{\mathrm{a}}$ & $12.21 \pm 0.08$ \\
\hline \multicolumn{5}{|c|}{ Selection Strategies $\times$ Close-bred Flocks } \\
\hline Pedigree $\times$ Major & $433.41 \pm 1.32^{\mathrm{a}}$ & $184.74 \pm 1.56^{\mathrm{a}}$ & $2.35 \pm 0.02^{c}$ & $12.17 \pm 0.16$ \\
\hline Pedigree $\times$ Kaleem & $433.52 \pm 1.29^{\mathrm{a}}$ & $183.35 \pm 1.70^{\mathrm{ab}}$ & $2.37 \pm 0.02^{\mathrm{bc}}$ & $11.99 \pm 0.11$ \\
\hline Pedigree $\times$ Saadat & $432.96 \pm 1.95^{\mathrm{a}}$ & $179.28 \pm 1.56^{\mathrm{bc}}$ & $2.42 \pm 0.02^{b}$ & $12.06 \pm 0.08$ \\
\hline Pedigree $\times$ Zahid & $432.63 \pm 1.61^{\mathrm{ab}}$ & $181.40 \pm 1.32^{\mathrm{abc}}$ & $2.39 \pm 0.02^{b c}$ & $12.26 \pm 0.16$ \\
\hline Mass Selection $\times$ Major & $429.56 \pm 0.86^{\mathrm{abcd}}$ & $181.16 \pm 1.11^{\mathrm{abc}}$ & $2.37 \pm 0.02^{\mathrm{bc}}$ & $12.36 \pm 0.22$ \\
\hline Mass Selection $\times$ Kaleem & $430.78 \pm 0.76^{\mathrm{abc}}$ & $181.05 \pm 0.94^{\mathrm{abc}}$ & $2.38 \pm 0.01^{\mathrm{bc}}$ & $12.19 \pm 0.11$ \\
\hline Mass Selection $\times$ Saadat & $426.89 \pm 1.26^{\mathrm{cd}}$ & $178.16 \pm 0.87^{\mathrm{cd}}$ & $2.40 \pm 0.02^{\mathrm{bc}}$ & $12.17 \pm 0.09$ \\
\hline Mass Selection $\times$ Zahid & $428.37 \pm 1.02^{\mathrm{bcd}}$ & $179.48 \pm 0.98^{\mathrm{bc}}$ & $2.39 \pm 0.01^{\mathrm{bc}}$ & $12.05 \pm 0.10$ \\
\hline Random-bred $\times$ Major & $425.81 \pm 1.22^{\mathrm{d}}$ & $172.00 \pm 1.25^{\mathrm{e}}$ & $2.48 \pm 0.02^{\mathrm{a}}$ & $12.36 \pm 0.15$ \\
\hline Random-bred $\times$ Kaleem & $430.89 \pm 1.20^{\mathrm{abc}}$ & $174.29 \pm 1.65^{\text {de }}$ & $2.48 \pm 0.03^{a}$ & $12.08 \pm 0.09$ \\
\hline Random-bred $\times$ Saadat & $431.11 \pm 1.20^{\mathrm{abc}}$ & $177.75 \pm 1.28^{\mathrm{cd}}$ & $2.43 \pm 0.02^{\mathrm{ab}}$ & $12.25 \pm 0.08$ \\
\hline Random-bred $\times$ Zahid & $429.63 \pm 2.32^{\text {abcd }}$ & $178.23 \pm 1.58^{\mathrm{cd}}$ & $2.42 \pm 0.02^{b}$ & $12.15 \pm 0.18$ \\
\hline \multicolumn{5}{|c|}{ Selection Strategies $\times$ Parental Age Groups } \\
\hline Pedigree $\times 10$ weeks & $434.31 \pm 1.55^{\mathrm{a}}$ & $185.99 \pm 1.44^{\mathrm{a}}$ & $2.34 \pm 0.02^{c}$ & $12.18 \pm 0.12$ \\
\hline Pedigree $\times 12$ weeks & $432.67 \pm 1.24^{\mathrm{ab}}$ & $181.37 \pm 1.05^{b}$ & $2.39 \pm 0.02^{\mathrm{bc}}$ & $12.22 \pm 0.13$ \\
\hline Pedigree $\times 14$ weeks & $432.42 \pm 1.21^{\mathrm{ab}}$ & $179.22 \pm 1.34^{\mathrm{bc}}$ & $2.42 \pm 0.02^{\mathrm{ab}}$ & $12.06 \pm 0.09$ \\
\hline Mass Selection $\times 10$ weeks & $428.36 \pm 0.84^{c}$ & $180.38 \pm 0.90^{b}$ & $2.38 \pm 0.01^{\mathrm{bc}}$ & $12.25 \pm 0.13$ \\
\hline Mass Selection $\times 12$ weeks & $428.28 \pm 0.97 c$ & $181.20 \pm 0.76^{b}$ & $2.37 \pm 0.01^{\mathrm{bc}}$ & $12.09 \pm 0.08$ \\
\hline Mass Selection $\times 14$ weeks & $430.06 \pm 0.83^{\mathrm{bc}}$ & $178.32 \pm 0.87^{\mathrm{bcd}}$ & $2.41 \pm 0.01^{\mathrm{ab}}$ & $12.23 \pm 0.14$ \\
\hline Random-bred $\times 10$ weeks & $429.94 \pm 0.93^{b c}$ & $176.06 \pm 1.31^{\mathrm{cd}}$ & $2.45 \pm 0.02^{\mathrm{a}}$ & $12.09 \pm 0.07$ \\
\hline Random-bred $\times 12$ weeks & $427.83 \pm 1.74^{c}$ & $175.63 \pm 1.30^{d}$ & $2.44 \pm 0.02^{\mathrm{a}}$ & $12.19 \pm 0.06$ \\
\hline Random-bred $\times 14$ weeks & $430.31 \pm 1.36^{b c}$ & $175.01 \pm 1.35^{d}$ & $2.46 \pm 0.02^{\mathrm{a}}$ & $12.35 \pm 0.17$ \\
\hline \multicolumn{5}{|c|}{ Close-bred Flocks × Parental Age Groups } \\
\hline Major $\times 10$ weeks & $430.93 \pm 1.33$ & $181.38 \pm 1.67^{\mathrm{ab}}$ & $2.38 \pm 0.02^{\mathrm{bcd}}$ & $12.31 \pm 0.20$ \\
\hline Major $\times 12$ weeks & $429.37 \pm 1.34$ & $180.87 \pm 1.64^{\mathrm{abc}}$ & $2.38 \pm 0.02^{\mathrm{cd}}$ & $12.17 \pm 0.08$ \\
\hline Major $\times 14$ weeks & $428.48 \pm 1.18$ & $175.66 \pm 1.51^{d}$ & $2.44 \pm 0.02^{\mathrm{ab}}$ & $12.41 \pm 0.22$ \\
\hline Kaleem $\times 10$ weeks & $432.19 \pm 1.12$ & $184.52 \pm 1.64^{a}$ & $2.35 \pm 0.02^{\mathrm{d}}$ & $12.07 \pm 0.10$ \\
\hline Kaleem $\times 12$ weeks & $431.26 \pm 0.91$ & $177.86 \pm 1.40^{\mathrm{bcd}}$ & $2.43 \pm 0.02^{\mathrm{abc}}$ & $12.04 \pm 0.12$ \\
\hline Kaleem $\times 14$ weeks & $431.74 \pm 1.33$ & $176.31 \pm 1.45^{\mathrm{cd}}$ & $2.45 \pm 0.02^{\mathrm{a}}$ & $12.14 \pm 0.09$ \\
\hline Saadat $\times 10$ weeks & $429.67 \pm 1.80$ & $178.21 \pm 1.12^{\mathrm{bcd}}$ & $2.41 \pm 0.02^{\mathrm{abc}}$ & $12.19 \pm 0.08$ \\
\hline Saadat $\times 12$ weeks & $428.85 \pm 1.58$ & $178.99 \pm 1.05^{\mathrm{bcd}}$ & $2.40 \pm 0.02^{\mathrm{abcd}}$ & $12.16 \pm 0.08$ \\
\hline Saadat $\times 14$ weeks & $432.44 \pm 1.25$ & $177.98 \pm 1.58^{\mathrm{bcd}}$ & $2.43 \pm 0.02^{\mathrm{abc}}$ & $12.12 \pm 0.09$ \\
\hline Zahid $\times 10$ weeks & $430.70 \pm 1.31$ & $179.14 \pm 1.78^{\mathrm{bcd}}$ & $2.41 \pm 0.02^{\mathrm{abc}}$ & $12.11 \pm 0.10$ \\
\hline Zahid $\times 12$ weeks & $428.89 \pm 2.31$ & $179.86 \pm 1.00^{\mathrm{bcd}}$ & $2.39 \pm 0.02^{\mathrm{bcd}}$ & $12.16 \pm 0.15$ \\
\hline Zahid $\times 14$ weeks & $431.04 \pm 1.50$ & $180.11 \pm 1.00^{\mathrm{bcd}}$ & $2.40 \pm 0.02^{\mathrm{abcd}}$ & $12.18 \pm 0.19$ \\
\hline
\end{tabular}

Note: Means with different superscripts in the same column are significantly different ( $p \leq 0.05)$; CBF: close-bred flocks; Cum: cumulative; Fl: feed intake; FCR: feed conversion ratio 
Ahmad S, Mehmood S, Javed K, Mahmud A, Usman M, Rehman A, Ishaq HM, Hussain J, Ghayas A

\section{Different Selection Strategies for the Improvement of the Growth Performance and Carcass Traits of Japanese Quails}

and mass-selected birds may be attributed to their higher nutrient requirements for growth. Consistently, in another study (Khaldari et al., 2010), higher feed intake was observed in Japanese quails selected for high body weight compared with the controls. In study with Japanese quails, significantly higher weekly feed intake differences were observed in mass-selected birds compared with the control group (Narinc \& Aksoy, 2012). In addition, Hussain et al. (2013) suggested that a selected line presented better feed efficiency than the control group. However, no significant effect of age on feed intake was also observed among three age groups of indigenous Peshawari Aseel chicken (Sohail et al., 2013).

Pedigree-base selected birds presented the highest body weight gain $(182.19 \pm 0.79 \mathrm{~g})$, followed by massselected birds $(179.96 \pm 0.50 \mathrm{~g})$ and random-bred controls $(175.57 \pm 0.76 \mathrm{~g})$. Among different parental age groups, birds from 10-week-old parents had higher weight gain $(180.81 \pm 0.81 \mathrm{~g})$ than those from 14-weekold parents $(177.52 \pm 0.71 \mathrm{~g})$. There were significant interactions $(p \leq 0.05)$ between selection strategies $\times$ parental age groups and selection strategies $\times$ CBF for cumulative weight gain. However, no significant effect of CBF on weight gain was observed in present study; however, CBF was compared among ages on overall basis significant $(p \leq 0.05)$ body weight differences were observed. Pedigree-base selected birds presented the highest body weight gain followed by mass-selected and random-bred controls, which may be due to the positive response to selection, differences in genetics of sub-strains of pedigree-based selected progenies, and the superior potential of pedigree birds selected for high body weight. The observed higher body weight may be the result of intense selection, which ultimately improves productive potential of birds. Pedigree birds showed better feed utilization, which resulted higher weight gain. Higher body weight in selected birds were also reported in other studies (Khaldari et al., 2010). Similarly, higher body weight gain was observed in Japanese quail lines selected for high body weight (Anthony et al., 1986). Among different parental age groups, the progeny of 10-week-old parents presented higher weight gain compared with the progeny of 14-week-old parents, which may be attributed to the fact that younger birds present higher weight gain potential compared with older ones. At different ages, significant body weight difference among strains and generations were also reported by Varkoohi et al, (2011). Similarly, in another study (Ahmad et al., 2013), a significant effect of age on body weight was observed in Mushki Aseel chickens.
In the present experiment, significantly $(p \leq 0.05)$ better FCR was observed in pedigree birds $(2.38 \pm 0.01)$ compared with mass-selected birds $(2.39 \pm 0.01)$ and random-bred controls (2.45 \pm 0.01$)$. Birds from 10 -week-oldparents showed better FCR $(2.39 \pm 0.01)$ compared with those from $12(2.40 \pm 0.01)$ and 14-week-old $(2.43 \pm 0.01)$ parents. In the present experiment, CBF had no effect on FCR; however, FCR was significantly affected $(p \leq 0.05)$ by the interactions between CBF and selection strategies and age groups. The interaction of selection strategies with parental age groups significantly influenced $(p \leq 0.05)$ cumulative FCR. However, no significant effect of selection methods, CBF or parental age groups on mortality \% was observed in the current experiment. In the present experiment, pedigree birds presented significantly better FCR compared with mass-selected and randombred control birds, which may be due to the lower maintenance requirements and lower fat deposition of pedigree-base selected birds. Usually, there is a favorable correlation between feed conversion ratio and growth because of enhanced pulsative growth hormone release and ultimately live weight gain. The selection for better feed conversion in broiler chickens resulted in direct selection for carcass leanness. Similarly, in another study (Marks, 1980), better FCR was observed in Japanese quails selected for high body weight for 42 generations compared with randombred controls. Birds from 10-week-old parents showed better FCR compared with the progenies of 12- and 14-w-oldparents, possibly because younger birds have better feed conversion ratio than older ones. However, Sohail et al. (2013) did not report any significant effect of age on the FCR of Peshawari Aseel chickens.

No significant cumulative mortality differences ( $p>$ 0.05 ) were observed among the different selection strategies, close-bred flocks, parental age groups or their interactions. However, other study reported significant effect of different selection strategies on the mortality of Japanese quails (Hussain et al., 2013), who argued that the lower mortality of intensively-selected birds resulted in the better exploitation of superior genes, and therefore, increased livability (Hussain et al., 2013).

\section{Carcass traits}

Means and standard errors of live body weight (g), carcass weight (g), and carcass yield (\%)are presented in Table 2. Significant live body weight ( $\mathrm{g}$ ) differences $(p \leq 0.05)$ were observed among the evaluated selection strategies. The progeny from pedigree-based selected 
Ahmad S, Mehmood S, Javed K,

Mahmud A, Usman M, Rehman A,

Ishaq HM, Hussain J, Ghayas A
Different Selection Strategies for the Improvement of the Growth Performance and Carcass Traits of Japanese Quails

Table 2 - Effect of selection strategies of parent Japanese quails from four close-bred flocks of three different age on the slaughter Parameters (mean \pm standard error) of their progenies.

\begin{tabular}{|c|c|c|c|}
\hline & $\begin{array}{l}\text { Live weight } \\
---(\mathrm{g})---\end{array}$ & $\begin{array}{c}\text { Carcass weight } \\
---(\mathrm{g})---\end{array}$ & $\begin{array}{c}\text { Carcass yield } \\
---(\%)---\end{array}$ \\
\hline \multicolumn{4}{|l|}{ Selection Strategies } \\
\hline Pedigree & $192.10 \pm 1.02^{\mathrm{a}}$ & $109.60 \pm 0.96^{a}$ & $57.07 \pm 0.42^{\mathrm{a}}$ \\
\hline Mass Selection & $185.35 \pm 0.50^{b}$ & $106.91 \pm 0.50^{b}$ & $57.70 \pm 0.29^{a}$ \\
\hline Random-bred & $181.45 \pm 0.67 c$ & $97.24 \pm 0.53^{c}$ & $53.63 \pm 0.33^{b}$ \\
\hline \multicolumn{4}{|l|}{ Close-bred Flocks } \\
\hline Major & $186.22 \pm 1.05$ & $104.47 \pm 1.04$ & $56.08 \pm 0.42$ \\
\hline Kaleem & $185.78 \pm 1.32$ & $104.68 \pm 1.19$ & $56.35 \pm 0.51$ \\
\hline Sadaat & $188.33 \pm 1.13$ & $104.96 \pm 1.26$ & $55.71 \pm 0.51$ \\
\hline Zahid & $184.87 \pm 0.79$ & $104.23 \pm 1.05$ & $56.38 \pm 0.49$ \\
\hline \multicolumn{4}{|l|}{ Parental Age Groups } \\
\hline 10 weeks & $185.53 \pm 0.87$ & $101.89 \pm 0.72^{b}$ & $54.93 \pm 0.35^{b}$ \\
\hline 12 weeks & $186.07 \pm 0.91$ & $103.18 \pm 0.57^{b}$ & $55.49 \pm 0.29^{b}$ \\
\hline 14 weeks & $187.30 \pm 1.06$ & $108.69 \pm 1.27^{a}$ & $57.97 \pm 0.48^{a}$ \\
\hline \multicolumn{4}{|c|}{ Selection Strategies $\times$ Close-bred Flocks } \\
\hline Pedigree $\times$ Major & $191.47 \pm 1.58^{\mathrm{ab}}$ & $109.13 \pm 1.49^{a}$ & $57.00 \pm 0.67^{a}$ \\
\hline Pedigree $\times$ Kaleem & $194.33 \pm 2.48^{\mathrm{a}}$ & $109.71 \pm 2.19^{a}$ & $56.50 \pm 1.01^{a}$ \\
\hline Pedigree $\times$ Sadaat & $194.13 \pm 2.41^{\mathrm{a}}$ & $110.56 \pm 2.38^{a}$ & $56.94 \pm 0.91^{\text {a }}$ \\
\hline Pedigree $\times$ Zahid & $188.47 \pm 1.28^{\mathrm{bc}}$ & $109.01 \pm 1.69^{a}$ & $57.83 \pm 0.74^{\mathrm{a}}$ \\
\hline Mass Selection $\times$ Major & $186.87 \pm 1.17^{\mathrm{cd}}$ & $107.02 \pm 1.05^{a}$ & $57.30 \pm 0.67^{a}$ \\
\hline Mass Selection $\times$ Kaleem & $183.47 \pm 0.68^{\text {def }}$ & $107.08 \pm 1.07^{\mathrm{a}}$ & $58.37 \pm 0.57^{a}$ \\
\hline Mass Selection $\times$ Sadaat & $186.80 \pm 1.08^{\mathrm{cd}}$ & $107.09 \pm 1.10^{\mathrm{a}}$ & $57.34 \pm 0.57^{a}$ \\
\hline Mass Selection $\times$ Zahid & $184.27 \pm 0.73^{\text {cde }}$ & $106.45 \pm 0.90^{\mathrm{a}}$ & $57.78 \pm 0.54^{\mathrm{a}}$ \\
\hline Random-bred $\times$ Major & $180.33 \pm 1.45^{\text {ef }}$ & $97.24 \pm 1.09^{b}$ & $53.94 \pm 0.55^{b}$ \\
\hline Random-bred $\times$ Kaleem & $179.53 \pm 1.16^{f}$ & $97.24 \pm 109^{b}$ & $54.19 \pm 0.66^{b}$ \\
\hline Random-bred $\times$ Sadaat & $184.07 \pm 1.04^{\text {cdef }}$ & $97.24 \pm 1.09 b$ & $52.85 \pm 0.62^{b}$ \\
\hline Random-bred $\times$ Zahid & $181.87 \pm 1.44^{\mathrm{ef}}$ & $97.24 \pm 1.09^{b}$ & $53.52 \pm 0.77^{b}$ \\
\hline \multicolumn{4}{|c|}{ Selection Strategies $\times$ Parental Age Groups } \\
\hline Pedigree $\times 10$ weeks & $189.15 \pm 1.61^{\mathrm{bc}}$ & $181.95 \pm 1.06^{c}$ & $55.72 \pm 0.50^{\mathrm{bc}}$ \\
\hline Pedigree $\times 12$ weeks & $192.10 \pm 1.54^{\mathrm{ab}}$ & $105.39 \pm 0.68^{c}$ & $54.93 \pm 0.54^{\text {cd }}$ \\
\hline Pedigree $\times 14$ weeks & $195.05 \pm 1.97^{a}$ & $118.12 \pm 1.38^{a}$ & $60.55 \pm 0.30^{a}$ \\
\hline Mass Selection $\times 10$ weeks & $185.95 \pm 1.16^{c d}$ & $105.24 \pm 0.72^{c}$ & $56.62 \pm 0.43^{b}$ \\
\hline Mass Selection $\times 12$ weeks & $185.20 \pm 0.71^{\mathrm{de}}$ & $104.97 \pm 0.63^{c}$ & $56.69 \pm 0.36^{b}$ \\
\hline Mass Selection $\times 14$ weeks & $184.90 \pm 0.66^{\mathrm{de}}$ & $110.51 \pm 0.65^{b}$ & $59.78 \pm 0.38^{a}$ \\
\hline Random-bred $\times 10$ weeks & $181.50 \pm 1.24^{\mathrm{ef}}$ & $95.12 \pm 0.34 e^{e}$ & $52.46 \pm 0.41^{e}$ \\
\hline Random-bred $\times 12$ weeks & $180.90 \pm 1.19^{f}$ & $99.17 \pm 0.93^{d}$ & $54.84 \pm 0.51^{\text {cd }}$ \\
\hline Random-bred $\times 14$ weeks & $181.95 \pm 1.06^{\mathrm{ef}}$ & $97.44 \pm 1.10^{\text {de }}$ & $53.58 \pm 0.64^{\text {de }}$ \\
\hline \multicolumn{4}{|c|}{ Close-bred Flocks $\times$ Parental Age Groups } \\
\hline Major $\times 10$ weeks & $187.87 \pm 1.78$ & $101.84 \pm 1.46^{d}$ & $54.19 \pm 0.48^{d}$ \\
\hline Major $\times 12$ weeks & $186.20 \pm 1.57$ & $103.50 \pm 1.23^{\mathrm{bcd}}$ & $55.57 \pm 0.37^{d}$ \\
\hline Major $\times 14$ weeks & $184.60 \pm 2.11$ & $108.06 \pm 2.27^{\mathrm{abc}}$ & $58.48 \pm 0.80^{\mathrm{a}}$ \\
\hline Kaleem $\times 10$ weeks & $183.80 \pm 2.46$ & $101.90 \pm 1.48^{d}$ & $55.52 \pm 0.86^{\text {bcd }}$ \\
\hline Kaleem $\times 12$ weeks & $185.60 \pm 2.11$ & $103.07 \pm 1.14^{\text {cd }}$ & $55.59 \pm 0.65^{\mathrm{bcd}}$ \\
\hline Kaleem $\times 14$ weeks & $187.93 \pm 2.29$ & $109.06 \pm 2.77^{\mathrm{ab}}$ & $57.95 \pm 0.98^{\mathrm{ab}}$ \\
\hline Sadaat $\times 10$ weeks & $186.33 \pm 1.18$ & $101.90 \pm 1.48^{d}$ & $54.69 \pm 0.73^{d}$ \\
\hline Sadaat $\times 12$ weeks & $188.00 \pm 1.90$ & $103.07 \pm 1.14^{\text {cd }}$ & $54.87 \pm 0.67^{d}$ \\
\hline Sadaat $\times 14$ weeks & $190.67 \pm 2.50$ & $109.92 \pm 2.95^{\mathrm{a}}$ & $57.56 \pm 1.04^{\mathrm{abc}}$ \\
\hline Zahid $\times 10$ weeks & $184.13 \pm 1.17$ & $101.90 \pm 1.48^{d}$ & $55.33 \pm 0.66^{\mathrm{cd}}$ \\
\hline Zahid $\times 12$ weeks & $184.47 \pm 1.71$ & $103.07 \pm 1.14^{\text {cd }}$ & $55.91 \pm 0.62^{\mathrm{bcd}}$ \\
\hline Zahid $\times 14$ weeks & $186.00 \pm 1.19$ & $107.72 \pm 2.34^{\text {abcd }}$ & $57.90 \pm 1.11^{\mathrm{ab}}$ \\
\hline
\end{tabular}

Note: - Means with different superscripts in the same column are significantly different $(p \leq 0.05)$ 
Ahmad S, Mehmood S, Javed K, Mahmud A, Usman M, Rehman A, Ishaq HM, Hussain J, Ghayas A

\section{Different Selection Strategies for the Improvement of the Growth Performance and Carcass Traits of Japanese Quails}

birds were the heaviest $(192.10 \pm 1.02 \mathrm{~g})$ followed by mass-selected $(185.35 \pm 0.50 \mathrm{~g})$ and random-bred control $(181.45 \pm 0.67 \mathrm{~g})$ progenies. The higher body weight of the pedigree-based selected birds might be attributed to the effect of continuous selection and to the positive relationship associated with higher fourweek body weight. The superior genes expressed in pedigree-based selected birds increased their live body weight. Higher body weight was reported in Japanese quails selected for high body weight were reported by Baylan et al. (2009). Higher pre-slaughter body weight was observed in Japanese quails selected for high four-week body weight for 19 generations (Dhaliwal et al., 2004). Regarding interaction between selection strategies and close-bred flocks and selection strategies and parental age groups significant differences live body weight were determined $(p \leq 0.05)$. However, different close-bred flocks and parental age groups as well as their interaction had no significant effect ( $p>0.05$ ) on progeny live body weight.

Significant differences $(p \leq 0.05)$ were observed in carcass weight (g) among progeny form different selection strategies and parental age groups. Pedigree-based selected bird also presented heavier carcasses $(109.60 \pm 0.96 \mathrm{~g})$ followed by massselected $(106.91 \pm 0.50 \mathrm{~g})$ and random-bred control $(97.24 \pm 0.53 \mathrm{~g})$ groups. The interactions among selection strategies, close-bred flocks and parental age groups significantly influenced $(p \leq 0.05)$ carcass weight. However, different close-bred flocks presented similar ( $p>0.05)$ carcass weight. The heavier carcasses of pedigree-based selected birds may be attributed to the positive correlation between body weight and carcass weight. Similarly, another study reported significant effects of selection on the carcass weight of Japanese quails selected for 11 generations (Alkanet al., 2010). Among different parental age groups, birds from 14-week-old parents presented highest carcass weight $(108.69 \pm 1.27 \mathrm{~g})$ compared with those from 10- $(103.18 \pm 0.57)$ and 12- $(101.89 \pm 0.72)$ weekold parents. Another study also observed significant effect of age on the carcass weight of Japanese quails (Tarhyel et al., 2012).

Significant carcass yield differences $(p \leq 0.05)$ were observed among different selection strategies and parental age groups. The progeny of selected birds (pedigree-based and mass-selected) presented higher $(57.07 \pm 0.42 \%, 57.70 \pm 0.29 \%)$ carcass yield compared with random-bred controls $(53.63 \pm 0.33 \%)$. However, no significant effect of selection on carcass yield was reported in Japanese quails selected for high 5-week body weight (Alkanet al., 2010). Among different parental age groups, the progeny of14-week-old parents presented higher $(57.97 \pm 0.48)$ carcass yield compared with those from $12-(55.49 \pm 0.29)$ and 10 $(54.93 \pm 0.35)$ week-old parents. A significant effect of age on carcass yield was also observed in Pharaoh quails by Wilkanowska \& Kokoszynski (2011). The interaction among selection strategies, close-bred flocks and parental age groups significantly influenced $(p \leq 0.05)$ carcass yield. However, non-significant differences $(p>$ 0.05 ) were observed among the different close-bred flocks.

Means and standard errors of cumulative relative weight (\%) of the liver, gizzard, and heart has been presented in Table 3. Significant liver relative weight differences $(p \leq 0.05)$ were observed among different selection strategies and parental age groups. Random-bred control birds presented the highest liver relative weight $(3.67 \pm 0.04 \%)$ followed by massselected $(3.19 \pm 0.02 \%)$ and pedigree-based selected $(3.08 \pm 0.02 \%)$ progenies. However, higher liver yield was observed in Japanese quails selected for high body weight (Ojedapo et al., 2008). Among the different parental age groups, the progeny of14week-old parents presented higher liver relative weight $(3.50 \pm 0.05 \%)$ followed by the progenies of 12 $(3.31 \pm 0.04)$ and $10-(3.14 \pm 0.02)$ week-old parents. A study with Japanese quails also found significant differences in liver relative weight at 3, 4 and 5 weeks of age (Akram et al., 2016). The interactions among selection strategies, close-bred flocks and parental age groups significantly influenced liver relative weight $(p \leq 0.05)$.

Significant differences $(p \leq 0.05)$ were observed in gizzard relative weight among different closebred flocks and parental age groups. Randombred control birds presented higher gizzard relative weight $(3.55 \pm 0.04 \%)$ compared with massselected $(3.17 \pm 0.03 \%)$ and pedigree-based selected $(3.09 \pm 0.03 \%)$ birds. This may be due to the negative association between carcass and gizzard weights, as birds selected for high body weight have proportionally lower gizzard relative weight. However, another study indicated higher gizzard weight \% in Japanese quails selected for high body weight (Abdel-Azeem et al., 2007). Among the different parental age groups, birds from 14-week-old parents presented higher gizzard relative weight $(3.49 \pm 0.04 \%)$ followed by the birds from $12(3.27 \pm 0.04 \%)$ and $10(3.05 \pm 0.02 \%)$ weekold parents. Similarly, another study revealed significant effect of age on gizzard relative weight in Japanese quails (Kaye et al., 2016). 
Ahmad S, Mehmood S, Javed K,

Mahmud A, Usman M, Rehman A,

Ishaq HM, Hussain J, Ghayas A
Different Selection Strategies for the Improvement of the Growth Performance and Carcass Traits of Japanese Quails

Table 3 - Effect of selection strategies of parent Japanese quails from four close-bred flocks of three different age giblet relative weights of their progenies.

\begin{tabular}{|c|c|c|c|}
\hline & Liver & Gizzard & Heart \\
\hline & \multicolumn{3}{|c|}{----------------(Mean\% \ Standard Error) ----------------- } \\
\hline \multicolumn{4}{|l|}{ Selection Strategies } \\
\hline Pedigree & $3.08 \pm 0.02^{c}$ & $3.09 \pm 0.03^{b}$ & $0.97 \pm 0.01^{c}$ \\
\hline Mass Selection & $3.19 \pm 0.02^{b}$ & $3.17 \pm 0.03^{b}$ & $1.01 \pm 0.01^{b}$ \\
\hline Random-bred & $3.67 \pm 0.04^{a}$ & $3.55 \pm 0.04^{a}$ & $1.03 \pm 0.01^{\mathrm{a}}$ \\
\hline \multicolumn{4}{|l|}{ Close-bred Flocks } \\
\hline Major & $3.30 \pm 0.05$ & $3.28 \pm 0.05$ & $1.01 \pm 0.01$ \\
\hline Kaleem & $3.31 \pm 0.05$ & $3.29 \pm 0.05$ & $1.00 \pm 0.01$ \\
\hline Sadaat & $3.29 \pm 0.05$ & $3.22 \pm 0.05$ & $0.99 \pm 0.01$ \\
\hline Zahid & $3.35 \pm 0.05$ & $3.30 \pm 0.05$ & $1.01 \pm 0.01$ \\
\hline \multicolumn{4}{|l|}{ Parental Age Groups } \\
\hline 10 weeks & $3.14 \pm 0.02^{c}$ & $3.05 \pm 0.02^{c}$ & $0.95 \pm 0.01 c$ \\
\hline 12 weeks & $3.31 \pm 0.04^{b}$ & $3.27 \pm 0.04^{b}$ & $1.00 \pm 0.01^{b}$ \\
\hline 14 weeks & $3.50 \pm 0.05^{a}$ & $3.49 \pm 0.04^{a}$ & $1.05 \pm 0.01^{\mathrm{a}}$ \\
\hline \multicolumn{4}{|c|}{ Selection Strategies $\times$ Close-bred Flocks } \\
\hline Pedigree $\times$ Major & $3.08 \pm 0.04^{\mathrm{bc}}$ & $3.09 \pm 0.08^{b}$ & $0.97 \pm 0.02^{\text {cde }}$ \\
\hline Pedigree $\times$ Kaleem & $3.03 \pm 0.05^{c}$ & $3.07 \pm 0.04^{b}$ & $0.96 \pm 0.02^{\text {de }}$ \\
\hline Pedigree $\times$ Sadaat & $3.04 \pm 0.03 c$ & $3.05 \pm 0.06^{b}$ & $0.96 \pm 0.01 \mathrm{e}$ \\
\hline Pedigree $\times$ Zahid & $3.17 \pm 0.04^{\mathrm{bc}}$ & $3.14 \pm 0.03^{b}$ & $0.99 \pm 0.01^{\text {bcde }}$ \\
\hline Mass Selection $\times$ Major & $3.18 \pm 0.04^{b c}$ & $3.15 \pm 0.04^{b}$ & $1.00 \pm 0.02^{\text {abcde }}$ \\
\hline Mass Selection $\times$ Kaleem & $3.21 \pm 0.03^{b}$ & $3.22 \pm 0.07^{b}$ & $1.02 \pm 0.01^{\mathrm{abc}}$ \\
\hline Mass Selection $\times$ Sadaat & $3.19 \pm 0.04^{b c}$ & $3.10 \pm 0.04^{b}$ & $1.00 \pm 0.02^{\text {abcde }}$ \\
\hline Mass Selection $\times$ Zahid & $3.20 \pm 0.04^{b c}$ & $3.21 \pm 0.08^{b}$ & $1.01 \pm 0.02^{\mathrm{abcd}}$ \\
\hline Random-bred $\times$ Major & $3.64 \pm 0.09^{a}$ & $3.59 \pm 0.09^{a}$ & $1.05 \pm 0.02^{\mathrm{a}}$ \\
\hline Random-bred $\times$ Kaleem & $3.69 \pm 0.06^{a}$ & $3.57 \pm 0.07^{\mathrm{a}}$ & $1.03 \pm 0.01^{\mathrm{ab}}$ \\
\hline Random-bred $\times$ Sadaat & $3.66 \pm 0.07^{a}$ & $3.50 \pm 0.08^{a}$ & $1.01 \pm 0.01^{\mathrm{abc}}$ \\
\hline Random-bred $\times$ Zahid & $3.70 \pm 0.07^{a}$ & $3.55 \pm 0.08^{a}$ & $1.03 \pm 0.02^{\mathrm{ab}}$ \\
\hline \multicolumn{4}{|c|}{ Selection Strategies $\times$ Parental Age Groups } \\
\hline Pedigree $\times 10$ weeks & $2.99 \pm 0.03^{f}$ & $2.96 \pm 0.03^{f}$ & $0.93 \pm 0.01^{e}$ \\
\hline Pedigree $\times 12$ weeks & $3.05 \pm 0.03^{\text {ef }}$ & $3.06 \pm 0.03^{e f}$ & $0.97 \pm 0.01^{\mathrm{cd}}$ \\
\hline Pedigree $\times 14$ weeks & $3.20 \pm 0.03^{d}$ & $3.24 \pm 0.06^{d}$ & $1.01 \pm 0.01^{b}$ \\
\hline Mass Selection $\times 10$ weeks & $3.08 \pm 0.02^{\mathrm{e}}$ & $2.97 \pm 0.02^{f}$ & $0.95 \pm 0.01^{\text {de }}$ \\
\hline Mass Selection $\times 12$ weeks & $3.16 \pm 0.01^{d}$ & $3.16 \pm 0.02^{\mathrm{de}}$ & $1.01 \pm 0.01^{b}$ \\
\hline Mass Selection $\times 14$ weeks & $3.35 \pm 0.02^{c}$ & $3.39 \pm 0.05^{c}$ & $1.07 \pm 0.01^{\mathrm{a}}$ \\
\hline Random-bred $\times 10$ weeks & $3.34 \pm 0.02^{c}$ & $3.21 \pm 0.03^{d}$ & $0.98 \pm 0.01 c$ \\
\hline Random-bred $\times 12$ weeks & $3.72 \pm 0.04^{b}$ & $3.60 \pm 0.04^{b}$ & $1.03 \pm 0.01^{b}$ \\
\hline Random-bred $\times 14$ weeks & $3.95 \pm 0.02^{\mathrm{a}}$ & $3.84 \pm 0.03^{a}$ & $1.08 \pm 0.01^{\mathrm{a}}$ \\
\hline \multicolumn{4}{|c|}{ Close-bred Flocks $\times$ Parental Age Groups } \\
\hline Major $\times 10$ weeks & $3.09 \pm 0.04^{d}$ & $3.01 \pm 0.04^{e}$ & $0.94 \pm 0.01^{f}$ \\
\hline Major $\times 12$ weeks & $3.28 \pm 0.08^{\mathrm{bcd}}$ & $3.28 \pm 0.08^{\mathrm{bcd}}$ & $1.01 \pm 0.01^{\mathrm{cd}}$ \\
\hline Major $\times 14$ weeks & $3.53 \pm 0.10^{\mathrm{a}}$ & $3.54 \pm 0.09^{a}$ & $1.07 \pm 0.01^{\mathrm{a}}$ \\
\hline Kaleem $\times 10$ weeks & $3.14 \pm 0.06^{c d}$ & $3.10 \pm 0.05^{\mathrm{de}}$ & $0.96 \pm 0.01^{\mathrm{ef}}$ \\
\hline Kaleem $\times 12$ weeks & $3.31 \pm 0.08^{\text {abcd }}$ & $3.28 \pm 0.07^{\mathrm{bcd}}$ & $1.00 \pm 0.01^{\mathrm{cd}}$ \\
\hline Kaleem $\times 14$ weeks & $3.48 \pm 0.09^{a b}$ & $3.48 \pm 0.09^{a b}$ & $1.05 \pm 0.01^{\mathrm{ab}}$ \\
\hline Sadaat $\times 10$ weeks & $3.15 \pm 0.04^{c d}$ & $3.01 \pm 0.04^{e}$ & $0.95 \pm 0.01^{\dagger}$ \\
\hline Sadaat $\times 12$ weeks & $3.29 \pm 0.09^{\mathrm{bcd}}$ & $3.21 \pm 0.07^{\text {cde }}$ & $0.99 \pm 0.01^{\text {de }}$ \\
\hline Sadaat $\times 14$ weeks & $3.44 \pm 0.10^{\mathrm{ab}}$ & $3.43 \pm 0.09^{a b}$ & $1.03 \pm 0.02^{b c}$ \\
\hline Zahid $\times 10$ weeks & $3.16 \pm 0.05^{\mathrm{cd}}$ & $3.07 \pm 0.04^{\mathrm{de}}$ & $0.96 \pm 0.01^{\mathrm{ef}}$ \\
\hline Zahid $\times 12$ weeks & $3.36 \pm 0.09 a b c$ & $3.33 \pm 0.08^{\mathrm{abc}}$ & $1.01 \pm 0.01^{\mathrm{cd}}$ \\
\hline Zahid $\times 14$ weeks & $3.54 \pm 0.07^{\mathrm{a}}$ & $3.50 \pm 0.07^{\mathrm{a}}$ & $1.06 \pm 0.01^{\mathrm{ab}}$ \\
\hline
\end{tabular}

Note: - Means with different superscripts in the same column are significantly different $(p \leq 0.05)$ 
Ahmad S, Mehmood S, Javed K, Mahmud A, Usman M, Rehman A, Ishaq HM, Hussain J, Ghayas A

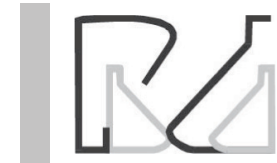

Significant differences $(p \leq 0.05)$ were observed in heart relative weight among different selection strategies and parental age groups. Randombred control birds had the highest heart relative weight $(1.03 \pm 0.01 \%)$, followed by mass-selected $(1.01 \pm 0.01 \%)$ and pedigree-based selected $(0.97 \pm 0.01 \%)$ progenies. Lower heart weight was also reported in commercial broilers selected for high body weight (Gaya et al., 2007; Tarhyel et al., 2012). Among different parental age groups, the progeny of14week-old parents showed the highest heart relative weight $(1.05 \pm 0.01 \%)$ followed by the progenies of 12 $(1 \pm 0.01 \%)$ and $10(0.95 \pm 0.01 \%)$ week-old parents. The results may be explained by the fact that, as birds age, body organs also follow the general body growth pattern to meet their anatomical and physiological demands. Consistently, significant differences in heart relative weight were observed among the progenies of different parental age groups of Japanese quails (Akram et al., 2013).

\section{CONCLUSIONS}

The results of the present study show that pedigreebased selection had pronounced effect on the growth performance and carcass traits of Japanese quails. In addition, it is suggested that parental ages of 10 and 14 weeks of age promote better progeny growth performance and carcass traits. The progenies of the different close-bred flocks did not present any differences in growth performance or carcass traits.

\section{ACKNOWLEDGEMENTS}

The contribution made by the administration of Avian Research and Training Centre, Department of Poultry Production, University of Veterinary and Animal Sciences, is highly appreciated.

\section{CONFLICT OF INTEREST}

No potential conflict of interest was found by the authors.

\section{REFERENCES}

Abdel-Azeem AS, Abdel-Azim AM, Darwish AA, Omar EM. Litter traits in four pure breeds of rabbits and their Crosses under prevailing environmental conditions of Egypt. Proceedings of the $5^{\text {th }}$ International Conference on Rabbit Production in Hot Climate; 2007; Hurghada. Egypt. p.39-51

Ahmad S, Hussain J, Akram M, Aslam F, Mahmud A, Mehmood S, et al. Comparative study on production performance and hatching traits of three age groups of indigenous Mushki Aseel chickens. Agriculture Advances 2013;2:146-149.
Different Selection Strategies for the Improvement of the Growth Performance and Carcass Traits of Japanese Quails

Akram M, Hussain J, Ahmad S, Mehmood S, Rehman A, Iqbal A, Usman M. Study of body measurements and slaughter characteristics in Japanese quail as influenced by age. Scientific Journal of Zoology 2013;2:23-26.

Alkan S, Karabag K, Galic A, Karsli T, Balcioglu MS. Effect of selection for body weight and egg production on egg quality traits in Japanese quail (Coturnix coturnix Japonica) of different lines and relationship between these traits. Kafkas University of Veterinary FakDerg 2010;16:277-280.

Anthony NB, Nestor KE, Bacon WL. Growth curves of Japanese quail as modified by divergent selection for 4-week body weight. Poultry Science 1986;65:1825-1833.

Ashok A, Prabakaran R. Effect of Non-Genetic Factors on Absolute Growth Rate in Short Term Selection for Different Ages in Japanese quail (Coturnix coturnix japonica). Journal of Livestock Science 2012;3:5256

Baylan M, Canogullari S, Sahin A, Copur G, Baylan M. Effect of different selection methods for body weight on some genetic parameters. Journal of Animal and Veterinary Advances 2009;8:1385-1391.

Burt D, Pourquie O. Genetics. Chicken genome - science nuggets to come soon. Science 2003;300(5626):1669.

Daikwo SI, Momoh OM, Dim NI. Heritability estimates of genetic and phenotypic correlations among some selected carcass traits of japanese quail (Coturnix coturnix japonica) raised in a sub-humid climate. Journal of Biology Agriculture \& Healthcare 2013;13:60-65.

Dhaliwal SK, Chaudhary ML, Brahand GS, Sandhu JS. Growth and carcass characteristics of selected and control lines of Japanese quails (Coturnix coturnix japonica). Indian Journal of Poultry Science 2004;39:112-119.

Durmuş İ, Alkan S, Narinç D, Karabağ K, Karsli T. Effects of mass selection on egg production on some reproductive traits in Japanese quail. European Poultry Science 2017;81:1-8.

Fuller RC, Baer CF, Travis J. How and when selection experiments might actually be useful. Integrated Comparative Biology 2005;45:391-404.

Gaya LG, Costa A, Ferraz JBS, Rezende FM, Mattos EC, Eler JP, et al. Genetic trends of absolute and relative heart weight in a male broiler line. Genetics Molecular Research 2007;6:1091-1096.

Hill WJ, Mackay TFC. Falconer DS and introduction to quantitative genetics Genetics 2004;167:1529-1536.

Hussain J, Akram M, Sahota AW, Javed K, Ahmad HA, Mehmood S, et al. Selection for higher three-week body weight in Japanese quail: 1. Effect on growth performance. The Journal of Animal \& Plant Sciences 2013;23(6):1496-1500

Jatoi AS, Sahota AW, Akram M, Javed K, Jaspal MH, Hussain J, et al. Effect of different body weight categories on the productive performance of four close-bred flocks of Japanese quails (Coturnix coturnix japonica). The Journal of Animal \& Plant Sciences 2013;23(1):7-13.

Kaye J, Akpa GN, Adeyinka IA, Nwagu BI. Estimates of genetic parameters for body weight and some economic important traits in the Japanese quails (Coturnix Coturnix Japonica). Researchjournali's Journal of Agriculture 2016;3(1):1-15.

Khaldari MA, Pakdel H, Yegane M, Javaremi NA, Berg P. Response to selection and genetic parameters of body and carcass weights in Japanese quail selected for 4-week body weight. Poultry Science 2010;89:1834-1841

Marks HL. Divergent selection for growth in Japanese quail under split and complete nutritional environment. 4. Genetic and correlated responses from generations 12 to 20 . Poultry Science 1991;70:453-462. 
Ahmad S, Mehmood S, Javed K,

Mahmud A, Usman M, Rehman A,

Ishaq HM, Hussain J, Ghayas A
Marks HL. Feed efficiency of selected and non-selected Japanese quail lines. Poultry Science 1980;59:6-10.

Marks HL. Long-term selection for body weight in Japanese quail under different environments. Poultry Science 1996;75:1198-1203.

Minvielle F, Monvoisin JL, Costa J, Frenot A. Quail lines selected on egg number either on pure line or on crossbred performance. Proceedings of the $12^{\text {th }}$ International Symposium on Current Problems in Avian Genetics (Aviagen); 1997; Pruhonice. Czech Republic.

Narinç D, Aksoy T, Kaplan S. Effects of multi-trait selection on phenotypic and genetic changes in japanese quail (Coturnix coturnix Japonica). The Journal of Poultry Science 2016;53:103-110.

Narinc D, Aksoy T, Karaman E, Aygun A, Firat MZ, Uslu MK. Japanese quail meat quality: Characteristics, heritabilities, and genetic correlations with some slaughter traits. Poultry Science 2013a;92(7):1735-1744.

Narinç D, Aksoy T. Effects of mass selection based on phenotype and early feed restriction on the performance and carcass characteristics in japanese quails. Kafkas Universitesi Veteriner Fakultesi Dergisi 2012;18:425-430

Narinc D, Aygun A, Sari T. Effects of cage type and mating ratio on fertility in japanese quails (Coturnix coturnix japonica) eggs. Agriculture Science Developments 2013b;2(1):4-7

Narinç D, Karaman E, Aksoy T. Effects of slaughter age and mass selection on slaughter and carcass characteristics in two lines of Japanese quail. Poultry Science 2014;93:762-769.

NRC - National Research Council. Nutrient requirement table of poultry. $9^{\text {th }}$ ed. Washington: National Academy Press; 1994

Odunsi AA, Kehinde MO. Influence of sex on carcass and organoleptic characteristics of meat from Japanese quails. Proceedings $14^{\text {th }}$ Annual Conference of Animal Science Nigeria; 2009; Ogbomoso. Nigeria. p.226-228.

Ojedapo LO, Akinokun O, Adedeji TA, Olayeni TB, Ameen SA, Amao SR. Effect of strain and sex on carcass characteristics of three commercial broilers reared on deep litter system in the derived savannah area of Nigeria. World Journal of Agriculture Science 2008;4:487-491.

\section{Different Selection Strategies for the Improvement of the Growth Performance and Carcass Traits of Japanese Quails}

Reddy RP. Symposium: the effects of long term selection on growth of poultry. Introduction. Poultry Science 1996;75:1164-1167.

SAS Institute. SAS® Users guide: statistics. Version 9.01. Cary; 2003/2004.

Sohail A, Muhammad A, Hussain J, Iqbal A, Usman M, Rehman A, et al. Comparative study on productive performance, egg quality, egg geometry, and hatching traits of three age groups of indigenous Peshawari Aseel chickens. Scientific Journal of Veterinary Advances 2013;2:21-25

Szwaczkowski T. Use of mixed model methodology in poultry breeding: estimation of genetic parameters. In: Muir WM, Aggrey SE, editor Poultry genetics, breeding and biotechnology. Wallingford: $C A B$ International; 2003. p. 165-210.

Tarhyel R, Henab SA, Tanimomo BK. Effects of age on organ weight and carcass characteristics of Japanese quail (Coturnix coturnix Japonica). Scientific Journal of Agriculture 2012;1:21-26.

Türkmut L, Altan O, Oğuz İ, Yalçin S. Effects of selection for four week body weight on slaughter, carcass, and abdominal fat and some organ weights and blood serum parameters in Japanese quail. Turkish Journal of Veterinary and Animal Sciences 1999;23(1):63-68.

Vali N, Edriss MA, Rahmani HR. Genetic parameters of body and some carcass trait in two quail strains. International Journal of Poultry Science 2005;4:296-300.

Varkoohi S, Babak MMS, Pakdel A, Javaremi AN, Zaghari M, Kause A. Response to selection for feed conversion ratio in Japanese quail. Poultry Science 2010;89:1590-1598.

Varkoohi S, Pakdel A, Babak MMS, Javaremi AN, Kause A, Zaghari M. Genetic parameters for feed utilization traits in Japanese quail. Poultry Science 2011;90:42-47.

Wilkanowska A, Kokoszynski D. Comparison of slaughter value in Pharaoh Quail of different ages. Journal of Central European Agriculture 2011;12:145-154 